\title{
Risk assessment in solitary fibrous tumors: validation and refinement of a risk stratification model
}

\author{
Elizabeth G Demicco ${ }^{1}$, Michael J Wagner ${ }^{2}$, Robert G Maki ${ }^{3,4}$, Vishal Gupta ${ }^{5}$, Ilya Iofin $^{6}$, \\ Alexander J Lazar ${ }^{7}$ and Wei-Lien Wang ${ }^{7}$ \\ ${ }^{1}$ Department of Pathology, Mount Sinai Hospital, New York, NY, USA; ${ }^{2}$ Cancer Medicine, The University of \\ Texas M. D. Anderson Cancer Center, Houston, TX, USA ${ }^{3}$ Monter Cancer Center, Northwell Health, New \\ Hyde Park, NY, USA; ${ }^{4}$ Cold Spring Harbor Laboratory, Cold Spring Harbor, NY, USA; ${ }^{5}$ Department of \\ Radiation Oncology, Mount Sinai Hospital, New York, NY, USA; ${ }^{6}$ Department of Surgery, Mount Sinai \\ Hospital, New York, NY, USA and ${ }^{7}$ Department of Pathology and Translational Molecular Pathology, The \\ University of Texas M. D. Anderson Cancer Center, Houston, TX, USA
}

\begin{abstract}
Solitary fibrous tumors are an uncommon sarcoma type characterized by NAB2-STAT6 gene fusion. While solitary fibrous tumors metastasize in $5-25 \%$ of cases, it has historically been challenging to determine which specific tumor and patient characteristics predict aggressive behavior. We previously reported on a novel risk stratification scheme for solitary fibrous tumors incorporating patient age, tumor size, and mitotic activity to predict risk of metastasis. Herein we validate this risk stratification scheme in an independent, lower-risk population of 79 patients with primary non-meningeal solitary fibrous tumors, and propose incorporating tumor necrosis as a fourth variable to further improve the risk score. Fifty-seven percent of cases were considered low risk, $29 \%$ intermediate risk, and $14 \%$ high risk for metastasis. Of 50 patients with sufficient clinical follow-up data, no metastases developed in the low-risk patients $(n=23)$, while there was a $7 \% 10$-year metastatic risk in the intermediate risk group $(n=17)$, and a $49 \% 5$-year metastatic risk for the high-risk patients $(n=10)$. When tumor necrosis was added as a fourth variable to the model, predictive power was enhanced. Under the revised stratification, the proportion of tumors identified as low risk increased to $66 \%$, with no metastasis at 10 years, intermediate risk cases comprised $24 \%$ with $10 \%$ risk of metastasis at 10 years, and high risk comprised $10 \%$ of cases with $73 \%$ risk of metastasis at 5 years. In Kaplan-Meier analysis, this fourth-variable stratification provided significant discrimination between the risk groups $(P=0.0005)$. These findings confirmed the clinical utility of our previously published risk stratification model and support the inclusion of necrosis as a fourth variable in the model.

Modern Pathology (2017) 30, 1433-1442; doi:10.1038/modpathol.2017.54; published online 21 July 2017
\end{abstract}

Solitary fibrous tumors are mesenchymal neoplasms showing fibroblastic differentiation, and characterized by a recurrent intra-chromosomal paracentric inversion involving the long arm of chromosome 12, resulting in NAB2-STAT6 gene fusion. ${ }^{1,2}$ They are known for having an indolent course, with relatively infrequent metastasis. ${ }^{3}$ Aside from cases, which have overt sarcomatous transformation (anaplasia/dedifferentiation) that tend to behave aggressively like

Correspondence: Dr EG Demicco, MD, PhD, Department of Pathology, The Mount Sinai Hospital, 1 Gustave L Levy Place, Box 1194, New York, NY 10025, USA,

E-mail: Elizabeth.Demicco@gmail.com

Received 28 January 2017; revised 16 March 2017; accepted 19

April 2017; published online 21 July 2017 other high-grade sarcomas, ${ }^{4}$ the behavior of solitary fibrous tumors has been difficult to predict, despite histopathologic criteria for malignancy proposed by England et al. ${ }^{5}$ in 1989. Unlike sarcomas such as leiomyosarcoma or undifferentiated pleomorphic sarcoma, conventional sarcoma grading schemes such as those devised by the Fédération Nationale des Centres de Lutte Contre le Cancer or National Cancer Institute have shown to be poorly applicable to solitary fibrous tumors. Moreover, sarcoma staging systems such as those incorporated into the recent American Joint Committee on Cancer staging manual (eighth edition) ${ }^{6}$ have not been validated for use in predicting the behavior of solitary fibrous tumors and appear to be better suited for traditional sarcomas. A better model for behavior and risk 
stratification in solitary fibrous tumors may be provided by the example of gastrointestinal stromal tumors (GIST), for which several competing risk stratification schemes exist, each with their own strengths and weaknesses in predicting tumor behavior. ${ }^{7}$ In keeping with the GIST model, in recent years a number of studies backed by more rigorous statistical analysis than the initial descriptive series have attempted to refine risk criteria and identify those which are most relevant in risk prediction in solitary fibrous tumors. ${ }^{8-12}$ Nevertheless, the reported findings are conflicting.

In our initial study of 103 patients with resected primary solitary fibrous tumors of non-meningeal sites, we re-evaluated reported histopathological factors and found that tumor size, age, and mitotic activity were prognostic for the development of metastasis, as was necrosis, but not cellularity or pleomorphism. ${ }^{9}$ In addition, we proposed a risk stratification model combining age, tumor size, and mitotic activity to predict patient outcomes, analogous to the systems commonly used for GIST. In contrast, the largest study of extra-thoracic, nonmeningeal solitary fibrous tumor $(n=243)$ reported that only mitotic activity, cellularity, and pleomorphism were adverse prognostic features, and quite counterintuitively, that large tumor size, was in fact a good prognostic feature. ${ }^{8}$ van Houdt et al. ${ }^{11}$ examined 81 patients and found that size and mitotic activity correlated with metastasis. A third series, which also included meningeal solitary fibrous tumor as well as extra- and intra-thoracic solitary fibrous tumor, found site and malignant histologic features (by the England criteria) to be predictors of outcome. ${ }^{10}$ It is important to note that different studies use different measures of outcome, including recurrence-free survival, disease-specific survival, metastasis-free survival, and overall survival, confounding the ability to directly compare these series.

Given the conflicting information in the literature, we sought to validate our risk stratification model in an independent cohort of solitary fibrous tumors from a different patient population from our original series, and if possible, further refine our model.

\section{Materials and methods}

\section{Patients and Tumor Tissues}

Upon Institutional Review Board approval, the pathology archives of Mount Sinai Hospital (Sinai) were searched for diagnoses of 'solitary fibrous tumor,' and 'hemangiopericytoma' diagnosed between 1996 and 2015. All available slides and blocks were retrieved and subjected to expert pathology review by EGD $(n=201)$. The electronic medical record and, where available, microfilm records were reviewed for clinical information on patient treatment and outcome (local recurrence, metastasis and survival). Cases not meeting diagnostic criteria for solitary fibrous tumor after expert review $(n=36)$ were excluded. Other exclusions included meningeal origin, recurrent or metastatic disease, cases with unknown tumor status, tumors that were not resected at Sinai, and patients with no data on primary tumor size or patient age. After all exclusions, the patient population included 82 patients with primary solitary fibrous tumor. Three of these patients presented with synchronous metastases and were also excluded from further analysis.

\section{Pathology Review}

For patients with multiple biopsies, or re-excisions after incomplete initial excision, tumor size was taken as the largest gross tumor measurement on the resected specimen. For five tumors resected piecemeal, the largest aggregate dimension was used, unless radiologic measurements were available. Patient age was calculated at date of tumor resection. Mitotic counts were performed in the most mitotically active area of the tumor. At least 50 high-power fields (HPF) were counted where tumor size and available slides allowed, and the single highest count per 10 consecutive fields used. For patients with multiple specimens (biopsy, resection, and re-resection) the highest count from any one specimen was used. Presence of tumor necrosis was scored as positive when involving $10 \%$ or more of the tumor, but hemorrhage or hyalinization was not tabulated. Cellularity and nuclear pleomorphism were scored as low, moderate, or high as previously defined. ${ }^{5}$ The presence of anaplasia/dedifferentiation was noted separately, if present.

\section{Risk Prediction Model}

Metastatic risk scores were calculated using our previously published model. ${ }^{9}$ Patient age was scored as 0 if $<55$ years and 1 if $\geq 55$ years. Mitotic activity was scored as 0 if $<1$ mitotic figure/10 HPF, 1 if $1-3$ mitotic figures/10 $\mathrm{HPF}$, or 2 if $\geq 4 / 10 \mathrm{HPF}$. Tumor size was scored as 0 if $<5 \mathrm{~cm}, 1$ if 5 to $<10 \mathrm{~cm}, 2$ if 10 to $<15 \mathrm{~cm}$, or 3 if $\geq 15 \mathrm{~cm}$ ). Total scores were summed, and scores of $0-2$ were considered low risk, 3-4 as intermediate risk, and 5-6 as high risk.

\section{Immunohistochemistry}

Immunohistochemical study for STAT6 was performed in a CLIA-approved clinical lab using antibody against the C-terminal of STAT6 (S-20, SC-621, 1:250 dilution; Santa Cruz, Dallas, TX, USA) using a Ventana autostainer (Ventana Medical Systems, Tucson, AZ, USA) on a previously constructed solitary fibrous tumor tissue microarray ${ }^{13}$ or on whole sections as part of routine diagnosis.

\section{Statistical Analysis}

Patient outcomes were calculated from date of primary resection, and were assessed using the 
Kaplan-Meier method, with significance evaluated using the log-rank test. Outcomes included risk of metastasis, in which death is censored, overall survival, and disease-specific survival. For outcomes, an alpha of $P<0.05$ was considered statistically significant. Where possible, univariable Cox proportional hazards models were used to determine prognostic significance of individual risk factors. Comparisons of continuous variables (age, mitotic count, tumor size, and risk sum) between anatomic sites was performed using analysis of variance testing with post hoc Tukey's test to compare individual sites. $P<0.01$ was considered significant to take into account multiple hypothesis testing. Comparisons of categorical variables were performed using the Fisher exact test.

To develop the modified risk stratification scheme, clinicopathologic and outcome data were retrieved from the original The University of Texas M. D. Anderson (MD Anderson) data set. ${ }^{9}$ Presence of tumor necrosis was scored as $0(<10 \%)$ or 1 $(\geq 10 \%)$ and added to the risk scores generated by summation of age, mitotic rate, and size scores. Receiver-operating characteristic (ROC) curves for risk score correlation with development of metastasis were generated to compare the model with and without inclusion of necrosis, and used to select optimum score cutoffs for low- and high-risk patients. The selected cutoff scores were then validated in the Sinai data set.

\section{Results}

Clinicopathologic features of 79 solitary fibrous tumors meeting criteria for study inclusion are presented in Table 1. Briefly, men comprised 53\% of the cohort. Median age at presentation was 59 years (range 5 months-88 years). The plurality of tumors $(46 \%)$ arose within the thorax, while head and neck was the second most common site, with $19 \%$ of cases, reflecting an active head and neck surgical service at our institution. In contrast to the initial series from MD Anderson, intra-abdominal tumors (including visceral, retroperitoneal, and pelvic sites) accounted for only $10 \%$. As such, the majority of tumors were small, with a median of $5.0 \mathrm{~cm}$. Similarly, mitotic activity was low. Over half of the cases $(57 \%)$ were scored as low risk, $29 \%$ as intermediate risk, and only $14 \%$ as high risk. Two cases were considered to have at least focal anaplasia/dedifferentiation. One of these was a high risk, $14 \mathrm{~cm}$ tumor with a $<2 \mathrm{~cm}$ focus of anaplasia, arising in the neck of an 86-year-old man, while the other was an intermediate risk $8.5 \mathrm{~cm}$ tumor with more extensive anaplasia arising in the chest wall of a 61-year-old man. STAT6 immunohistochemistry was available in 68 cases, and was positive in 62 (91\%). Histologic review confirmed the diagnosis in negative cases; in most cases loss of staining was consistent with decreased antigenicity in old tissue blocks, as previously described. ${ }^{13}$

There were some significant differences in tumors based on site (Table 1). Tumors arising within the abdominal cavity were significantly larger (median $11.7 \mathrm{~cm}$ ) than tumors of the head and neck and trunk (median $4.2 \mathrm{~cm}, P=0.0004$ and $4.0 \mathrm{~cm}, P=0.008$, respectively), while intra-abdominal and pleural tumors arose at an older age than tumors of the trunk (median 71 and 64, respectively, vs 44 years, $P=0.005)$. Mitotic activity was similar regardless of site. Risk scores were higher in intra-abdominal tumors relative to head and neck, extremity, or trunk $(P<0.005)$. Although size and mitotic activity were shown to be independent risk factors in our prior series, mitotic score and tumor size did correlate to some extent. All tumors $\geq 10 \mathrm{~cm}$ had some degree of mitotic activity (14 with 1-3 mitotic figures/10 HPF $(61 \%)$, and 9 with $\geq 4$ mitotic figures/10 $\mathrm{HPF}(39 \%)$, compared to 12 tumors smaller than $10 \mathrm{~cm}$ without mitosis (22\%), 31 with 1-3 (56\%), and 12 (22\%) with $\geq 4$ mitotic figures/10 $\mathrm{HPF}, P=0.02$ ). Presence of necrosis was also more frequent in larger and more mitotically active tumors $(P=0.0001$ and 0.023 , respectively), just as it was in the original MD Anderson data set $(P=0.0001$ and 0.0026, respectively).

\section{Treatment and Outcomes}

All patients underwent primary surgical resection with curative intent. Clinical follow-up was available in 50 patients with median time of 44 months (interquartile range (IQR) 15-81 months). Two patients underwent re-resection within 2 months of initial surgery due to positive margins, and one patient was treated with post-operative radiation for positive margins. One patient received pre-operative radiation. One patient with an intra-abdominal tumor received neo-adjuvant chemotherapy, but developed local recurrence after resection; the tumor subsequently metastasized locally throughout the abdominal cavity. An additional three patients developed metastatic disease only (within the pleural cavity, to bone, or to lung and liver). Risk of metastasis was calculated to be $13 \%$ at 5 and 10 years (Figure 1 ). The dedifferentiated/anaplastic tumor arising in the chest wall was among those cases that metastasized, while the tumor with focal anaplasia in the neck had not recurred at 4 months follow-up.

In Kaplan-Meier analysis, mitotic activity of $\geq 4 / 10 \mathrm{HPF}$ was correlated with risk of metastasis $(P=0.027)$, as was necrosis $(P=0.0023)$, and tumor size $\geq 15 \mathrm{~cm}(P=0.0041)$. Age $\geq 55$, high cellularity, and nuclear pleomorphism were not significant. In univariate Cox proportional hazards models, high mitotic activity trended to predict metastasis (HR 8.5, 95\% CI 0.9-81.8, $P=0.065$ ), while size $\geq 15 \mathrm{~cm}$ was an adverse risk factor (HR 13.1, 95\% CI 1.4126.9, $P=0.026)$. Although a higher proportion of 


\section{Table 1 Clinicopathologic data}

\begin{tabular}{|c|c|c|c|c|c|c|c|}
\hline & \multirow{2}{*}{ All } & \multirow{2}{*}{$\begin{array}{l}\text { Cases with clinical } \\
\text { follow-up only }\end{array}$} & \multicolumn{5}{|c|}{ Site } \\
\hline & & & Intra-thoracic & Head and neck & Trunk & Extremity & $\begin{array}{l}\text { Intra- } \\
\text { abdominal }\end{array}$ \\
\hline Total & 79 & 50 & 36 & 15 & 11 & 9 & 8 \\
\hline M & 42 & 24 & 20 & 8 & 6 & 3 & 5 \\
\hline $\mathrm{F}$ & 37 & 26 & 16 & 7 & 5 & 6 & 3 \\
\hline $\begin{array}{l}\text { Age at presentation, median } \\
\text { (range) }\end{array}$ & $\begin{array}{l}59 \text { years } \\
\text { ( } 5 \text { months-88 } \\
\text { years) }\end{array}$ & $\begin{array}{l}61 \text { years } \\
(5 \text { months-88 years) }\end{array}$ & $\begin{array}{l}64 \text { years } \\
(31-82 \text { years })\end{array}$ & $\begin{array}{l}52 \text { years } \\
\text { (5 months-88 years) }\end{array}$ & $\begin{array}{l}44 \text { years } \\
\text { (26-63 years) }\end{array}$ & $\begin{array}{l}47 \text { years } \\
\text { (33-81 years) }\end{array}$ & $\begin{array}{l}71 \text { years } \\
\text { (46-88 years) }\end{array}$ \\
\hline \multicolumn{8}{|l|}{ Site, n (\%) } \\
\hline Intra-thoracic & $36(46)$ & $20(40)$ & & & & & \\
\hline Head and neck & $15(19)$ & $9(18)$ & & & & & \\
\hline Trunk & 11 (14) & $8(16)$ & & & & & \\
\hline Extremity & $9(11)$ & $5(10)$ & & & & & \\
\hline Intra-abdominal & $8(10)$ & $8(16)$ & & & & & \\
\hline \multicolumn{8}{|l|}{ Risk class } \\
\hline Low & $45(57 \%)$ & $23(46 \%)$ & $18(50 \%)$ & $13(87 \%)$ & $8(73 \%)$ & $6(67 \%)$ & 0 \\
\hline Moderate & $23(29 \%)$ & $17(34 \%)$ & $12(33 \%)$ & $1(7 \%)$ & $3(27 \%)$ & $3(33 \%)$ & $4(50 \%)$ \\
\hline High & $11(14 \%)$ & $10(20 \%)$ & $6(17 \%)$ & $1(7 \%)$ & 0 & 0 & $4(50 \%)$ \\
\hline Tumor size, median (range) & $5.0 \mathrm{~cm}(0.4-30)$ & $6.5 \mathrm{~cm}(1-30)$ & $5.8 \mathrm{~cm}(1-23)$ & $4.2 \mathrm{~cm}(0.4-14)$ & $4 \mathrm{~cm}(1.8-15.5)$ & $5 \mathrm{~cm}(2-10)$ & $11.7 \mathrm{~cm}(7-30)$ \\
\hline $\begin{array}{l}\text { Mitotic figures/10 HPF, } \\
\text { median (range) }\end{array}$ & $2(0-102)$ & $3(0-102)$ & $2(0-33)$ & $2(0-17)$ & $2(0-102)$ & $2(0-5)$ & $4(2-17)$ \\
\hline Necrosis & $15(19 \%)$ & $14(28 \%)$ & $6(17 \%)$ & 0 & $3(27 \%)$ & $1(11 \%)$ & $5(63 \%)$ \\
\hline Local recurrence & & $1(2 \%)$ & 0 & 0 & 0 & 0 & 1 \\
\hline Metastasis & & $4(8 \%)$ & 1 & 0 & 1 & 0 & 2 \\
\hline \multicolumn{8}{|l|}{ Metastatic sites } \\
\hline Peritoneum & & 1 & & & & & 1 \\
\hline Pleura & & 1 & 1 & & & & \\
\hline Lung & & $1^{\mathrm{a}}$ & & & & & $1^{\mathrm{a}}$ \\
\hline Bone & & 1 & & & 1 & & \\
\hline Liver & & $1^{\mathrm{a}}$ & & & & & $1^{\mathrm{a}}$ \\
\hline
\end{tabular}

asame patient. 
clinically aggressive tumors arose in extra-thoracic soft tissue $(3 / 30,10 \%$ vs $1 / 20,5 \%)$, this difference was not statistically significant; site did not provide additional prognostic information in Kaplan-Meier analysis.

Survival follow-up was available for 62 patients with median time of 46 months (IQR 15-87 months). Estimated 5- and 10-year overall survivals were 88\% and $76 \%$, respectively (Figure 1). Of eight deaths, only one was definitely attributable to metastatic solitary fibrous tumor, while three were from other causes and four were of unknown etiology in patients who were lost to follow-up.

\section{Validation of the Metastatic Risk Score}

Clinical follow-up data were available for 23 low-risk tumors, 17 moderate-risk, and 10 high-risk solitary fibrous tumors. No metastases were seen in low-risk

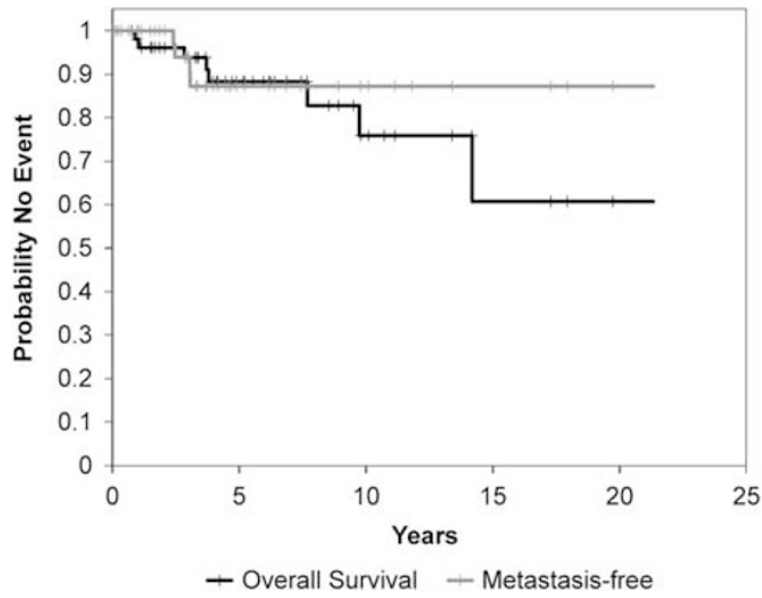

Figure 1 Clinical outcomes for 50 solitary fibrous tumors with follow-up. Kaplan-Meier plot shows overall survival and time to first metastasis. cases during the course of follow-up (median 36 months, IQR 8-96 months), while moderate-risk patients had a $7 \%$ risk of metastasis at 5 and 10 years. In contrast, high-risk solitary fibrous tumors had a $49 \%$ risk of metastasis at 5 years (Figure 2a). This difference was statistically significant; log-rank $P=0.014$. No statistically significant differences in overall survival were present based on risk score $(P=0.34$; Figure 2b).

\section{Refinement of the Metastatic Risk Score}

Although we were unable to show that necrosis was an independent risk factor for development of metastasis in our previous series due to lack of adequate sample size for full multivariable Cox proportional hazards models, necrosis was a significant risk factor in univariate analyses in both that series and in the current data set. We therefore inquired whether addition of tumor necrosis to the risk stratification model would better delineate benign from malignant tumors. To test this, we returned to our previously published data set and calculated new risk sums with the inclusion of $\geq 10 \%$ tumor necrosis as a fourth variable. We calculated sensitivity and specificity for history of metastasis at each cutoff score (Table 2), and generated ROC curves comparing the original threevariable risk stratification model with the fourvariable model (Figure 3). The area under the curve was similar for both the three-variable model (0.906) and four-variable model (0.901), indicating their relative comparability and strong association with metastatic risk.

As in the original model, low risk was defined as the range of scores in which no metastasis occurred, and high risk was defined as having at least $80 \%$ specificity for malignancy ( $\leq 20 \%$ false positive rate).
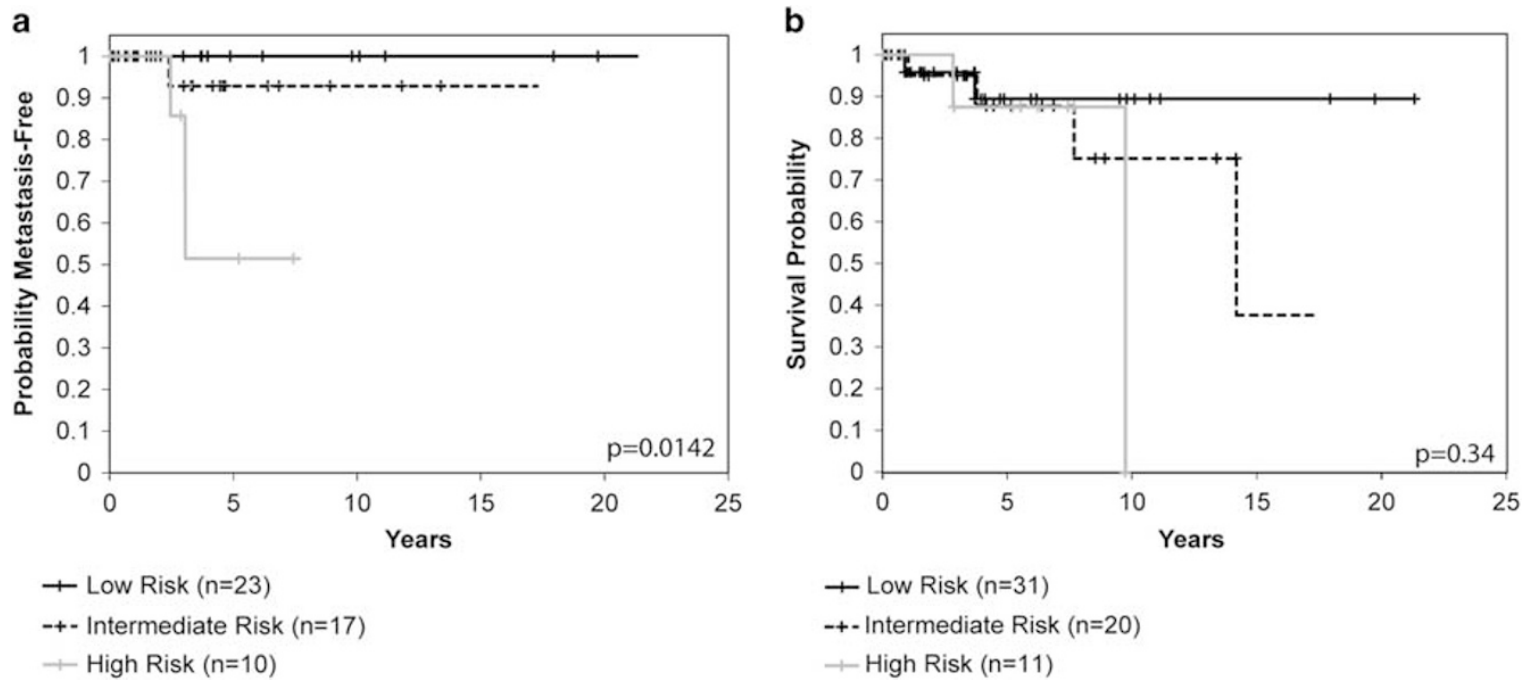

Figure 2 Validation of the three-variable risk stratification score. (a) Kaplan-Meier plot for time to metastasis by risk group. (b) KaplanMeier plot for overall survival. 
Table 2 Predicted sensitivity and specificity of original and modified risk stratification models for development of metastasis in solitary fibrous tumors

\begin{tabular}{cccccc}
\hline & \multicolumn{2}{c}{ Sensitivity } & & \multicolumn{2}{c}{ Specificity } \\
\cline { 2 - 3 } \cline { 5 - 6 } Sum & $\begin{array}{c}\text { Three- } \\
\text { variable } \\
\text { model }\end{array}$ & $\begin{array}{c}\text { Four- } \\
\text { variable } \\
\text { model }\end{array}$ & & $\begin{array}{c}\text { Three- } \\
\text { variable } \\
\text { model }\end{array}$ & $\begin{array}{c}\text { Four- } \\
\text { variable } \\
\text { model }\end{array}$ \\
\hline 0 & 1 & 1 & & 0.02 & 0.02 \\
1 & 1 & 1 & & 0.17 & 0.16 \\
2 & 1 & 1 & & 0.44 & 0.40 \\
3 & 0.95 & 1 & & 0.65 & 0.59 \\
4 & 0.74 & 0.89 & & 0.86 & 0.71 \\
5 & 0.47 & 0.58 & & 1 & 0.94 \\
6 & 0.00 & 0.37 & & 1 & 1 \\
7 & - & 0 & & - & 1 \\
\hline
\end{tabular}

In the initial study, low risk was defined as cutoff score having a sensitivity of $100 \%$ (all malignant tumors falling above the cutoff) and high risk as having a $100 \%$ specificity. Using the same criteria for the modified score, low risk includes scores $0-3$ and high risk 6-7.

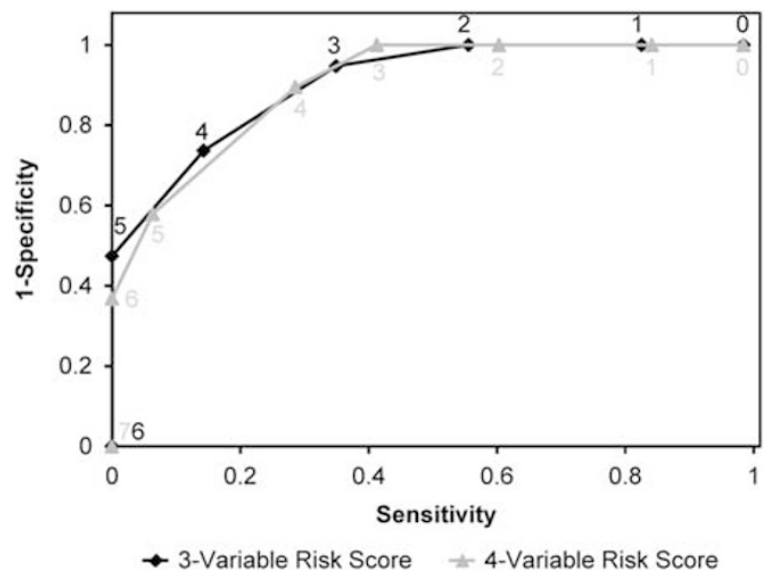

Figure 3 ROC curves for three- and four-variable risk stratification scores. Numbers indicate the risk scores for each plotted data point. Area under curve for three-variable score is 0.906. Area under curve for four-variable score is 0.901 .

In the new four-variable model, a risk score of $0-3$ was defined as low risk and a score of $6-7$ as high risk, while scores of 4-5 were considered as intermediate risk (Table 3). By adding necrosis to the model, $37(45 \%)$ patients in the test set were considered low risk, with no risk of metastasis at 510 years, while $30(37 \%)$ fell into the intermediate risk group, with $31 \%$ and $50 \%$ 5- and 10-year metastatic risk, respectively. The high-risk group comprised 15 patients (18\%) with 100\% risk of metastasis by 5 years (Table 4). In Kaplan-Meier analysis (Figure 4a), this difference was statistically significant (log-rank $P<0.0001$ ).

We then applied this four-variable model to our validation set of patients, and found that 52 patients $(66 \%)$ would now be considered low risk, $19(24 \%)$ intermediate risk, and 8 (10\%) high risk. Among the 50 patients with clinical follow-up, 28 low-risk patients
Table 3 Modified four-variable risk stratification model for development of metastasis in solitary fibrous tumors

\begin{tabular}{lc}
\hline Risk factor & Score \\
\hline $\begin{array}{l}\text { Age } \\
\quad<55\end{array}$ & 0 \\
$\quad \geq 55$ & 1 \\
& \\
Tumor size (cm) & 0 \\
$\quad<5$ & 1 \\
5 to $<10$ & 2 \\
10 to $<15$ & 3 \\
$\geq 15$ & \\
Mitotic count (/10 high-power fields) & 0 \\
0 & 1 \\
$1-3$ & 2 \\
$\geq 4$ & \\
Tumor necrosis & 0 \\
$\quad<10 \%$ & 1 \\
$\geq 10 \%$ & Total score \\
Risk class & $0-3$ \\
\hline Low & $4-5$ \\
Intermediate & $6-7$ \\
\hline High & \\
\hline
\end{tabular}

had no risk of metastasis at 5-10 years, the 15 intermediate-risk cases had only a $10 \%$ risk of metastasis at 5 and 10 years, and the 7 patients in the high-risk class had a $73 \%$ risk of metastasis at 5 years (Table 4 ). In Kaplan-Meier analysis, this refined risk stratification system was significantly associated with differences in metastatic risk (log-rank $P=0.0005$; Figure $4 \mathrm{~b}$ ).

Sensitivity and specificity of the risk scores for malignancy in the validation set were similar to the initial series (Table 5), with the exception that in the validation set there were no malignant tumors in the group of cases with modified risk score of 4 . The addition of necrosis as a fourth variable therefore increased the percentage of patients correctly defined as low risk, and more precisely identified a high-risk group with rapid development of metastasis.

While the four-variable model was also correlated with differences in overall survival and diseasespecific survival in the test set (log-rank $P=0.0002$ and $P<0.0001$, respectively, data not shown), there was only a trend to associate with disease-specific survival in the validation set (log-rank $P=0.054$, with only one death attributable to metastatic solitary fibrous tumor), and no statistically significant association with overall survival (log-rank $P=0.3$ ).

\section{Comparability of Risk Score Calculated on Core Biopsy and Resection Specimens}

We compared mitotic counts and necrosis scores between core biopsy and subsequent primary resection specimen for 32 cases among the initial and validation series for which both core biopsy 
Table 4 Risk class sizes and relative outcomes of original and modified risk stratification models

\begin{tabular}{|c|c|c|c|c|c|c|c|}
\hline \multirow{3}{*}{ Risk group } & \multirow{3}{*}{ Score } & \multicolumn{3}{|c|}{ Test set $(\mathrm{n}=82)$} & \multicolumn{3}{|c|}{ Validation set $(\mathrm{n}=50)$} \\
\hline & & \multirow{2}{*}{$\mathrm{N}(\%)$} & \multicolumn{2}{|c|}{ Metastasis-free } & \multirow{2}{*}{$\mathrm{N}(\%)$} & \multicolumn{2}{|c|}{ Metastasis-free } \\
\hline & & & 5 years & 10 years & & 5 years & 10 years \\
\hline \multicolumn{8}{|l|}{ Three-variable model } \\
\hline Low risk & $0-1$ & $28(34)$ & $100 \%$ & $100 \%$ & $23(46)$ & $100 \%$ & $100 \%$ \\
\hline Intermediate risk & $3-4$ & $31(38)$ & $77 \%$ & $64 \%$ & $17(34)$ & $93 \%$ & $93 \%$ \\
\hline High risk & $5-6$ & $23(28)$ & $15 \%$ & $0 \%$ & $10(20)$ & $49 \%$ & - \\
\hline \multicolumn{8}{|l|}{ Four-variable model } \\
\hline Low risk & $0-3$ & $37(45)$ & $100 \%$ & $100 \%$ & $28(56)$ & $100 \%$ & $100 \%$ \\
\hline Intermediate risk & $4-5$ & $30(37)$ & $69 \%$ & $50 \%$ & $15(30)$ & $90 \%$ & $90 \%$ \\
\hline High risk & $6-7$ & 15 (18) & $0 \%$ & $0 \%$ & $7(14)$ & $27 \%$ & - \\
\hline
\end{tabular}

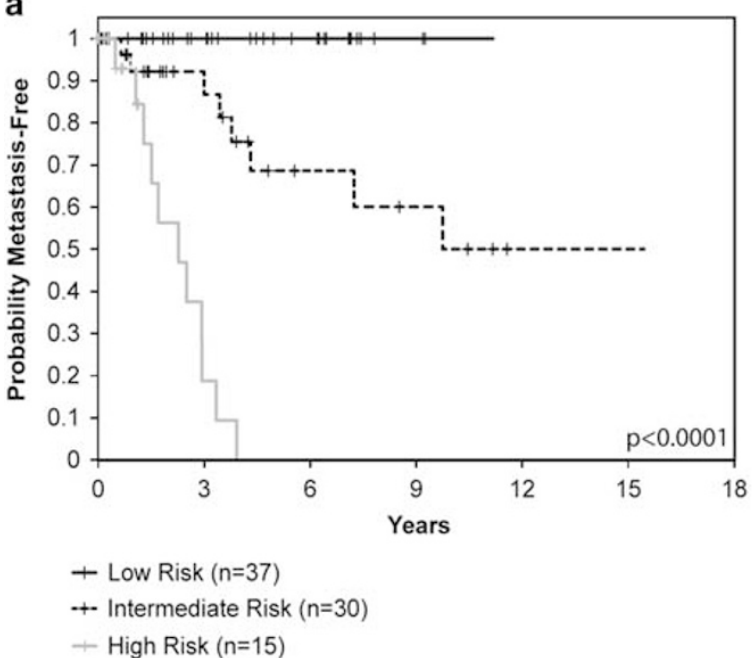

b

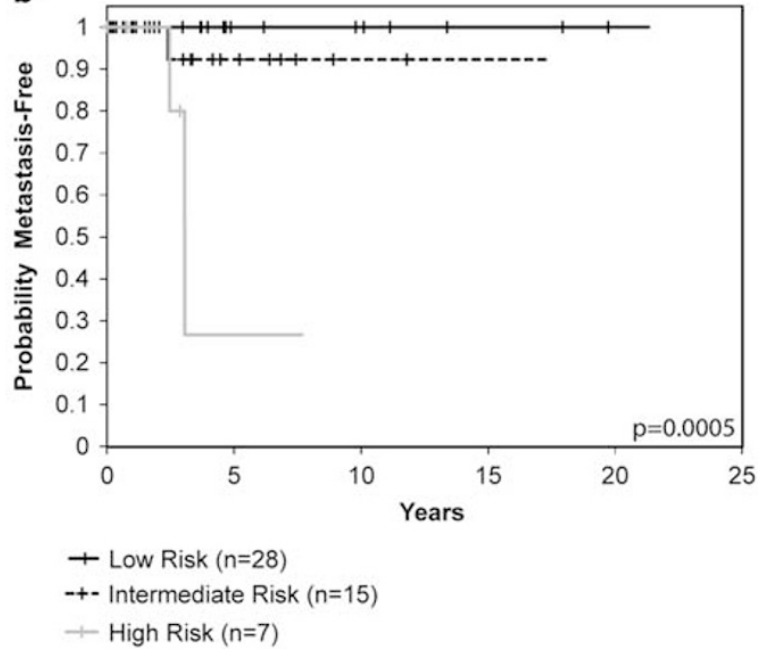

Figure 4 The modified four-variable risk stratification score is significantly associated with metastatic risk in both the test and validation populations. (a) Kaplan-Meier plot for four-variable risk stratification score and time to metastasis in the 82 patients in the test set. (b) Kaplan-Meier plot for the four-variable risk stratification score and time to metastasis in the 50 patients in the validation set.

specimens and resection specimens were available. Mitotic counts were lower in 20 of the core biopsies compared to the resection (63\%), and necrosis showed discrepant scoring in 10 biopsies compared to resection specimens (31\%). In 9 of 30 paired cases $(30 \%)$ for which risk score was calculated, these discrepancies resulted in lower final risk score in the core biopsy relative to the resection (5 low risk on biopsy/intermediate risk on resection, and 4 intermediate risk on biopsy/high risk on resection). In 5 cases, the core biopsy showed greater mitotic activity or necrosis than the resection, but the risk score remained the same on resection.

\section{Discussion}

Our findings successfully validate our previously published risk assessment score for solitary fibrous tumors, which was developed specifically to predict metastatic risk in solitary fibrous tumor. ${ }^{9}$ We furthermore present and validate a refinement on the model that incorporates the presence of tumor necrosis into the risk stratification scheme, resulting in improved ability to identify solitary fibrous tumors at low risk for metastasis, and more precisely delineate the patients most at risk for aggressive behavior.

Our validation patient population was different in a number of ways from our initial data set. The previous MD Anderson cohort was comprised of similar numbers of pleural and intra-abdominal tumors, with relatively fewer head and neck, extremity, and trunk solitary fibrous tumors, while the present series was largely composed of pleural solitary fibrous tumors, with head and neck representing the second most frequent site. Moreover, the tumors in the present series were smaller than in the initial series, in particular pleural tumors (median 15 
Table 5 Sensitivity and specificity of original and modified risk stratification models for development of metastasis in the validation series of solitary fibrous tumors

\begin{tabular}{lccccc}
\hline & \multicolumn{2}{c}{ Sensitivity } & & \multicolumn{2}{c}{ Specificity } \\
\cline { 2 - 3 } \cline { 5 - 6 } Sum & $\begin{array}{c}\text { Three- } \\
\text { variable } \\
\text { model }\end{array}$ & $\begin{array}{c}\text { Four- } \\
\text { variable } \\
\text { model }\end{array}$ & & $\begin{array}{c}\text { Three- } \\
\text { variable } \\
\text { model }\end{array}$ & $\begin{array}{c}\text { Four- } \\
\text { variable } \\
\text { model }\end{array}$ \\
\hline 0 & 1 & 1 & & 0.00 & 0.00 \\
1 & 1 & 1 & & 0.22 & 0.22 \\
2 & 1 & 1 & & 0.50 & 0.50 \\
3 & 1 & 1 & & 0.65 & 0.61 \\
4 & 0.75 & 1 & & 0.85 & 0.74 \\
5 & 0.50 & 0.75 & & 0.98 & 0.91 \\
6 & 0 & 0.50 & & 1 & 1 \\
7 & - & 0 & & - & 1 \\
\hline
\end{tabular}

vs $5.8 \mathrm{~cm}$ ) and extremity solitary fibrous tumors (median 8.7 vs $5 \mathrm{~cm}$ ). These differences likely reflect the position of MD Anderson as a tertiary cancer referral center, handling more complex and advanced cases than typically present to a primary hospital such as Mount Sinai. On the other hand, patients in the validation series were older, with intra-abdominal tumors in particular presenting at a much more advanced age in the present series (71 vs 51 years). Tumors in the present series were in general less aggressive than our initial series, with a lower rate of metastasis, and only one case with local recurrence. Our validation set most likely is a more accurate reflection of the biology of solitary fibrous tumors seen at most institutions, as our metastatic rate is similar to that seen in several other series, ${ }^{10,14-16}$ while the higher incidence of metastasis in our original series is similar to that reported at other tertiary referral centers. ${ }^{11}$

The present cohort is somewhat smaller than our original series, with limited follow-up available, particularly for low-risk tumors, though it is still sizable considering the difficulty in generating large data sets of rare mesenchymal tumors in singleinstitution series. Despite these limitations, our risk score was able to identify those cases at risk of metastasis with $100 \%$ sensitivity, with all metastatic tumors falling into moderate- and high-risk categories, and no metastases developing in low-risk cases. Thus, our findings show that despite variations in clinical settings and patient populations, our risk score provides reproducible results based on simple, relatively objective criteria, which are easy to identify from pathology reports and clinical data.

It must be emphasized that this risk score varies from most other solitary fibrous tumor studies in that it was specifically designed to assess metastatic risk/malignant potential, and is not intended to predict local recurrence, defined as tumor recurrence in or adjacent to the tumor bed/site of prior excision. In neither of our series were local recurrences frequent, and local recurrences were usually thought to be due to incomplete resection, rather than any underlying tumor biology.
Unfortunately, the contribution of margin status to local recurrence risk could not be definitely proven in either of our data sets due to incomplete clinical data and infrequent local recurrence.

This risk score also is not validated for prediction of survival outcomes. While both our three- and fourvariable model were associated with disease-specific survival in the test population, there was only one death attributable to disease in the validation series and we were unable to confirm this correlation. Given the older age at which solitary tumors typically arise, and the relative indolence of even metastatic tumors, patients often die of other causes before tumor recurrence, or even after tumor recurrence.

A caveat of our risk model is that it is designed and validated using primary tumors, and ideally intended for use on resection specimens. As with other sarcoma risk assessment and grading schemes, evaluation of core biopsies may result in inappropriately lower risk scores, due to sampling bias. ${ }^{17-19}$ In our experience, the variability between biopsy and resection did not result in any change to the risk score for patients with tumors, which were otherwise low risk (tumor $<10 \mathrm{~cm}$ and age $<55$ years), and one could be more confident in applying the risk model to core biopsies in this scenario. However, in larger tumors in older patients, low mitotic counts and absence of necrosis on core biopsy should be interpreted with caution, and such results might best be interpreted as the minimal or 'at least' level of risk given the potential for underestimation. Full application of the risk stratification system would be reserved for carefully sampled resection specimens.

Three recent series have emphasized tumor cellularity and nuclear pleomorphism as prognostic features; $8,10,11$ results we have not been able to replicate-with the exception of anaplastic solitary fibrous tumor, which also typically have high mitotic rates and often are of large size, as were the two cases in our series. While we agree that anaplasia is likely a risk factor for aggressive behavior, the subjective nature of determinations of cellularity and pleomorphism render them poorly reproducible and as such, we do not recommend relying upon them for routine patient care.

Our initial model did not incorporate the presence of tumor necrosis as a risk factor due to our inability to prove that it was an independent risk separate from tumor size and mitotic activity, two variables with which it was significantly correlated. However, necrosis was a strong prognostic factor in univariable analyses, and we revisited the question of whether or not addition of necrosis to the model could provide additional prognostic information. Indeed, we found that the inclusion of necrosis as a variable in our risk model allowed us to better predict both benign (nonmetastatic) and malignant behavior. This new model was also validated using our independent data set.

Importantly, our revised model takes into account multiple interrelated aspects of tumor histology. Traditionally, mitotic activity of $\geq 4 / 10 \mathrm{HPF}$ has 
been the single most frequent criteria used to define malignancy in solitary fibrous tumors. Our data consistently show that mitotic activity alone is not enough to accurately discriminate aggressive tumors, and is just one factor in the assessment equation. Moreover, while our original three-variable model is effective in identifying a majority of benign tumors, it too showed that it could be refined by the inclusion of additional variables to result in criteria that more narrowly define malignancy in solitary fibrous tumor. As yet, our classification scheme only takes into account readily identifiable clinicopathologic features, but does not address molecular factors driving the behavior of solitary fibrous tumor. Initial studies of NAB2-STAT6 fusion types had suggested that the specific exons present in the chimeric fusion gene might influence solitary fibrous tumor behavior, with $N A B 2$ exon 4-STAT6 exon 2 or 3 fusions thought to be less aggressive than $N A B 2$ exon 6STAT6 exon 16-18 fusions, ${ }^{20}$ but subsequent studies have not been as supportive of this finding. ${ }^{21,22}$ More recently, TERT promoter mutations, reported to be present in $\sim 22 \%$ of solitary fibrous tumors ${ }^{14,23-26}$ have emerged as another potential driver of aggressive behavior in solitary fibrous tumor, ${ }^{14,23}$ but the initial series have been small and require validation.

While the hope for molecular predictors of behavior for solitary fibrous tumor remains, the fact is that routine molecular analyses for prognostication of rare tumors remain out of reach for many practices. It is therefore important to develop simple reproducible guidelines for risk assessment in solitary fibrous tumors to provide appropriate clinical guidance. The ability to reliably predict aggressive behavior in solitary fibrous tumors would allow the majority of patients to be reassured of a curative surgical procedure, while truly high-risk patients could receive appropriate counseling and follow-up. Our modified risk stratification model fulfills these criteria, being both easy to use, and having reproducible results as proven in this validation series, despite the marked differences in the patient population between the initial and present studies.

\section{Acknowledgments}

We thank the Biorepository and Pathology Core at the Icahn School of Medicine at Mount Sinai for specimen retrieval, histology services, and generation of the tissue microarray.

\section{Disclosure/conflict of interest}

The authors declare no conflict of interest.

\section{References}

1 Chmielecki J, Crago AM, Rosenberg M, et al. Wholeexome sequencing identifies a recurrent NAB2-STAT6 fusion in solitary fibrous tumors. Nat Genet 2013;45: 131-132.

2 Robinson DR, Wu YM, Kalyana-Sundaram S, et al. Identification of recurrent NAB2-STAT6 gene fusions in solitary fibrous tumor by integrative sequencing. Nat Genet 2013;45:180-185.

3 Fletcher CDM, Bridge JA, Lee J-C. Extrapleural solitary fibrous tumor. In: Fletcher CDM, Bridge JA, Hogendoorn PCW (eds). World Health Organization of Tumors of Soft Tissue and Bone. IARC Press: Lyon, France, 2013, pp 80-82.

4 Mosquera JM, Fletcher CD. Expanding the spectrum of malignant progression in solitary fibrous tumors: a study of 8 cases with a discrete anaplastic componentis this dedifferentiated SFT? Am J Surg Pathol 2009;33: 1314-1321.

5 England DM, Hochholzer L, McCarthy MJ. Localized benign and malignant fibrous tumors of the pleura. A clinicopathologic review of 223 cases. Am J Surg Pathol 1989;13:640-658.

6 Amin MB, Edge S, Greene F, et al. (eds). AJCC Cancer Staging Manual, 8th edn. American Joint Committee on Cancer: Chicago, IL, USA, 2017.

7 Agaimy A. Gastrointestinal stromal tumors (GIST) from risk stratification systems to the new TNM proposal: more questions than answers? A review emphasizing the need for a standardized GIST reporting. Int J Clin Exp Pathol 2010;3:461-471.

8 Pasquali S, Gronchi A, Strauss D, et al. Resectable extra-pleural and extra-meningeal solitary fibrous tumours: a multi-centre prognostic study. Eur J Surg Oncol 2016;42:1064-1070.

9 Demicco EG, Park MS, Araujo DM, et al. Solitary fibrous tumor: a clinicopathological study of 110 cases and proposed risk assessment model. Mod Pathol 2012;25:1298-1306.

10 Wilky BA, Montgomery EA, Guzzetta AA, Ahuja N, Meyer CF. Extrathoracic location and "borderline" histology are associated with recurrence of solitary fibrous tumors after surgical resection. Ann Surg Oncol 2013;20:4080-4089.

11 van Houdt WJ, Westerveld CM, Vrijenhoek JE, et al. Prognosis of solitary fibrous tumors: a multicenter study. Ann Surg Oncol 2013;20:4090-4095.

12 Doyle LA, Fletcher CD. Predicting behavior of solitary fibrous tumor: are we getting closer to more accurate risk assessment? Ann Surg Oncol 2013;20: 4055-4056.

13 Demicco EG, Harms PW, Patel RM, et al. Extensive survey of STAT6 expression in a large series of mesenchymal tumors. Am J Clin Pathol 2015;143: 672-682.

14 Bahrami A, Lee S, Schaefer IM, et al. TERT promoter mutations and prognosis in solitary fibrous tumor. Mod Pathol 2016;29:1511-1522.

15 Vallat-Decouvelaere AV, Dry SM, Fletcher CD. Atypical and malignant solitary fibrous tumors in extrathoracic locations: evidence of their comparability to intra-thoracic tumors. Am J Surg Pathol 1998;22: 1501-1511.

16 Gold JS, Antonescu CR, Hajdu C, et al. Clinicopathologic correlates of solitary fibrous tumors. Cancer 2002;94:1057-1068.

17 Khoja H, Griffin A, Dickson B, et al. Sampling modality influences the predictive value of grading in adult soft tissue extremity sarcomas. Arch Pathol Lab Med 2013;137:1774-1779. 
18 Hoeber I, Spillane AJ, Fisher C, Thomas JM. Accuracy of biopsy techniques for limb and limb girdle soft tissue tumors. Ann Surg Oncol 2001;8:80-87.

19 Strauss DC, Qureshi YA, Hayes AJ, et al. The role of core needle biopsy in the diagnosis of suspected soft tissue tumours. J Surg Oncol 2010;102:523-529.

20 Barthelmess S, Geddert H, Boltze C, et al. Solitary fibrous tumors/hemangiopericytomas with different variants of the NAB2-STAT6 gene fusion are characterized by specific histomorphology and distinct clinicopathological features. Am J Pathol 2014;184: 1209-1218.

21 Tai HC, Chuang IC, Chen TC, et al. NAB2-STAT6 fusion types account for clinicopathological variations in solitary fibrous tumors. Mod Pathol 2015;28: 1324-1335.

22 Huang SC, Li CF, Kao YC, et al. The clinicopathological significance of NAB2-STAT6 gene fusions in 52 cases of intrathoracic solitary fibrous tumors. Cancer Med 2016;5:159-168.

23 Akaike K, Kurisaki-Arakawa A, Hara K, et al. Distinct clinicopathological features of NAB2-STAT6 fusion gene variants in solitary fibrous tumor with emphasis on the acquisition of highly malignant potential. Hum Pathol 2015;46:347-356.

24 Liau JY, Lee JC, Tsai JH, et al. Comprehensive screening of alternative lengthening of telomeres phenotype and loss of ATRX expression in sarcomas. Mod Pathol 2015;28:1545-1554.

25 Koelsche C, Renner M, Hartmann W, et al. TERT promoter hotspot mutations are recurrent in myxoid liposarcomas but rare in other soft tissue sarcoma entities. J Exp Clin Cancer Res 2014;33:33.

26 Koelsche C, Sahm F, Capper D, et al. Distribution of TERT promoter mutations in pediatric and adult tumors of the nervous system. Acta Neuropathol 2013;126:907-915. 